\title{
Sensing in the Mouth: A Model for Filiform Papillae as Strain Amplifiers
}

\author{
Eric Lauga ${ }^{1}$, Christopher J. Pipe ${ }^{2}$ and Benjamin Le Révérend ${ }^{2 *}$ \\ ${ }^{1}$ Department of Applied Mathematics and Theoretical Physics, University of Cambridge, Cambridge, UK, ${ }^{2}$ Nestlé Research \\ Center, Lausanne, Switzerland
}

Texture perception of foods is a common yet remarkably unstudied biophysical problem. Motivated by recent experiments reporting the presence of corpuscular endings in tongue filiform papillae, we develop in this work a mechanical model of the human tongue covered with filiform papillae in the form of elastic beams. Considering the typical flows that occur in the mouth during oral evaluation of Newtonian liquids, we suggest that filiform papillae may act either as direct strain sensors and/or as indirect strain amplifiers for the underlying mucosal tissue. Application of this model may also be valid for other biological appendages, such as primary cilliae and superficial neuromasts.

Keywords: texture perception, soft matter mechanics, sensory biophysics, neuromasts, primary cilliae, papillae

\section{OPEN ACCESS}

Edited by:

Antonio F. Miguel,

University of Évora, Portugal

Reviewed by:

Andrew Resnick

Cleveland State University, USA

Charles Wolgemuth,

University of Arizona, USA

*Correspondence:

Benjamin Le Révérend benjamin.lereverend@rdls.nestle.com

Specialty section:

This article was submitted to Interdisciplinary Physics,

a section of the journal

Frontiers in Physics

Received: 10 May 2016

Accepted: 26 July 2016

Published: 23 August 2016

Citation:

Lauga E, Pipe CJ and Le Révérend $B$ (2016) Sensing in the Mouth: A Model for Filiform Papillae as Strain Amplifiers. Front. Phys. 4:35. doi: 10.3389/fphy.2016.00035

\section{INTRODUCTION}

Food perception is based on the interaction between the human physiology and physical and chemical characteristics of food [1]. Molecular scale interactions between chemical compounds and receptors are usually responsible for taste, aroma and chemesthesis. Taste and chemesthesis pertain to the tongue [2-4] and the cells hosting taste receptors are located in onion-like structures called taste buds, located in circumvallate, foliate, and fungiform papillae. A fourth type of papilla, filiform papillae, are devoid of taste buds and are believed to be involved in texture perception, but the details of both the mechanical and molecular aspects of filiform papillae in encoding food texture are still unknown.

One important avenue of research consists in deciphering how the topological features of the tongue, mainly covered by filiform papillae, support the biological function of texture perception of foods-originally coined as psycho-rheology [5]. The particularly high sensitivity of the tongue in detecting small changes in stresses applied to the tissue remains to be conclusively linked to a physiological mechanism. Low yet perceptible stress changes can proceed from viscosity changes due to enzymatic [6], mechanical [7], or thermal breakdown of food structures, or due to the presence of micron-sized rigid particles in an otherwise homogeneous visco-elastic fluid such as glass beads in yogurt [8] or more universally a grain of sand in an oyster.

In this work, motivated by recent experiments reporting the presence of corpuscular endings in filiform papillae of mice tongues [9], we make the assumption that the origin of the high tactile acuity of the human tongue is the sum of mechanosensitive innervation [10], favorable transmission due to tissue mechanical properties and surface topology. Structures of similar aspect ratio to filiform papillae used to sense external fluid stresses have already been described in the animal kingdom. Theses include primary cilia at the cellular level [11] or superficial neuromasts in the fish lateral line $[12,13]$. To function in a similar manner filiform papillae would need to bend significantly under typical in-mouth flows. Supporting the latter, we derive a coupled bio-fluid mechanical model of the mechanical advantage lying in the presence of filiform papillae. 
This study is organized as follows. In Section 2 we introduce our modeling assumptions, including the geometry of papillae and mouth, their mechanical characteristics, the sensory function of the tongue and further physical assumptions. In Section 3 we then describe the flow as the tongue is moving and derive the scalings for the deformation strains of the papillae induced by the viscous fluid forces in Section 4. The scalings for the resulting elastic deformation of the tongue due to the bending of the clamped papillae are derived in Section 5 where we show that papillae are able to act as strain amplifiers by more than two orders of magnitude.

\section{MODEL}

\subsection{Morphology}

Over the last 60 years, a number of groups have investigated the morphology of filiform papillae (oftentimes motivated with associated diseases). Detailed studies exist on rats [14, 15], mice [16], porcupine [17], wild boar and pig [18], and humans [19, 20]. An overview of evolution in papilla morphology among vertebrates was detailed in Iwasaki [21]. An overview of the literature indicates the following morphological characteristics. In small vertebrates, individual papillae have a typical width ranging from 15 to $20 \mu \mathrm{m}$, and often taper conically at their tip. This width corresponds to the size of two to three epithelial cells, and is about the same order as the typical distance between papillae on the tongue. Their length can vary greatly between the anterior and posterior parts of the tongue and range from 50 to $100 \mu \mathrm{m}$. A precise set of measurements for humans were reported in Yamashita and OdDalkhsuren [22] where a distinction is made between the wide papillae body, which is anchored in the tongue, and the hairs on the papillae which do protrude upward from the tongue and deform under flow. Their size is reported to be $34 \pm 16 \mu \mathrm{m}$ in width and and $250 \pm 62 \mu \mathrm{m}$ long. Other measurements reports significantly larger sizes, with widths that can range $100-300 \mu \mathrm{m}$ and length $200-500 \mu \mathrm{m}$ $[21,23,24]$. Given the wide range of measured values among all the gathered papers, we will consider a half-width (radius) of $50 \mu \mathrm{m}$ and length $250 \mu \mathrm{m}$ in this paper as representative numbers.

\subsection{Mechanics}

No measurements of the Young's modulus of filiform papillae is available in the literature. A measure of $E=15 \mathrm{kPa}$ of the pig's tongue modulus was obtain on fresh pig tongues [24]. That number in the range which would have been obtained by focusing solely on the elasticity of epithelial cells, for example $E \approx 1-10 \mathrm{kPa}$ for monkey kidney epithelial cells [25] while $E \approx 0.1-300 \mathrm{kPa}$ for human foreskin epithelial cells, with an average of $14 \mathrm{kPa}$ for young cells and $33 \mathrm{kPa}$ for old cells [26]. In light of these published data, we will use a value of $E=25 \mathrm{kPa}$ in this study.

\subsection{Mathematical Model}

Based on the previous section, we can now put forward the model considered in this work, illustrated in Figure 1. We consider the fluid-solid interactions in a model mouth. The palate is modeled as a smooth, rigid, flat surface, at a distance $H$ from the tongue. The tongue is assumed to be a smooth, rigid, flat surface on which filiform papillae are distributed. The total length of the flow region from the anterior to the posterior side of the mouth is denoted $W$. The focus of the work is on the deformation of the filiform papillae. Each papilla is modeled as a straight elastic rod of length $L$, radius $a$, clamped on the tongue. The Young's modulus of the rod is denoted $E$. As discussed above, we take $E \approx 25 \mathrm{kPa}$ as a representative value. We also assume $a=50 \mu \mathrm{m}$, leading to a bending modulus $B=\pi a^{4} E / 4 \approx 1.2 \times 10^{-13} \mathrm{~J} . \mathrm{m}$.

\subsection{Sensory Function of the Tongue}

As stated in the introduction, the bovine tongue sensory innervation has already been partially described [10]. In addition to this, a recent study carried out in mice reported the presence of corpuscular endings innervating each filiform papillae [9], confirming a similar finding from previous work also describing innervation of filiform papillae in cats [27]. Since those endings do not project to the surface of the oral epithelium, we can hypothesize that they are mechanosensitive in nature and do

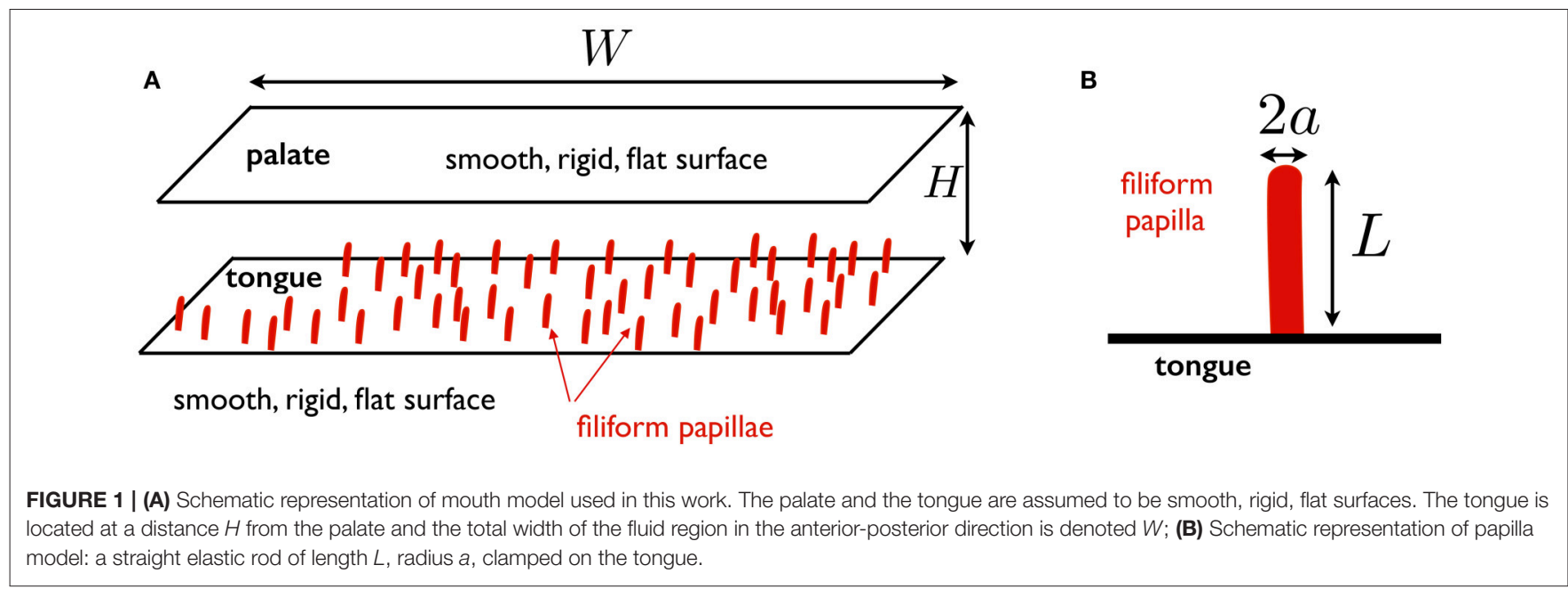


not pertain to chemical stimulation. Our motivation to study the deformation mechanics of filiform papillae under flow lies in the fact that applied macroscopic strains will cascade into microscopic cell and transmembrane protein deformations and lead to somatosensory sensing as already known in the mamallian skin [28]. This should thus open a window for biophysicists regarding the levels of stresses that are likely to be applied to mechanosensory cells when mammals probe food for its texture.

\section{FLOW INDUCED BY THE MOTION OF THE TONGUE}

\subsection{Parameters}

As we are interested in the interactions between deforming papillae and the flow of the food product, it is important to accurately model the driving of the flow between the palate and the tongue. During tasting and swallowing the palate is stationary and the tongue moves horizontally parallel to and vertically toward the palate driven by the action of lingual muscles. The typical setup is therefore that of (1) a squeeze flow driven by the vertical motion of the bottom surface (so-called transverse motion in the literature [29]) and (2) a shear component also present during tasting (referred to as longitudinal motion [29]). The shear component of the tongue movement can play an important role when acting alone, but as we see below the stresses it creates can safely be neglected when a transverse flow is present.

The study is motivated by the measurements of textures on complex fluids such as ice cream. Rheological measurements for $38 \%$ fat cream show a viscosity of about $20 \mathrm{mPa}$.s, hence approximately 20 times that of water [30]. Ice-creams typically have a viscosity in the range 20-300 times that of water [31], with strong variation with temperature and fat content. Recent measurements for ice cream with fat, fat replacers, and sweeteners indeed confirm this showing viscosities $O(100 \mathrm{mPa} . \mathrm{s})$ $[32,33]$. In this study we pick a reference viscosity of $\mu=$ $100 \mathrm{mPa} . \mathrm{s}$, equivalent to 100 times that of water at room tenperature.

It is also necessary to account for typical velocities in mouth during food consumption. Detailed measurements of movement in the mouth during swallowing in show velocities ranging from 1 to $15 \mathrm{~cm} / \mathrm{s}$, with an average around $10 \mathrm{~cm} / \mathrm{s}$ Shawker et al. [29]. This is consistent with results from computational simulations [34]. For a tongue-palate distance of $H \approx 5 \mathrm{~mm}$, a papilla size $L \approx 250 \mu \mathrm{m}$, and a viscosity $\mu=100 \mathrm{mPa}$.s, this leads to Reynolds numbers $O(1-10)$ in the mouth while the flows around the papillae are characterized by $\operatorname{Re} \sim 10^{-1}$ or even smaller due to the no-slip boundary condition near the papillae. Given that the relevant Reynolds number in narrow geometries is the regular Reynolds number times the channel aspect ratio, we are in the low-Reynolds number regime and can neglect the influence of inertia on the dynamics of the fluid in the mouth $[35,36]$.

\subsection{Newtonian Fluid Flow}

For the flow in the mouth we consider the basic setup illustrated in Figure 2. The tongue is assumed to move both in the longitudinal (horizontal) direction, with a typical velocity $U$, and

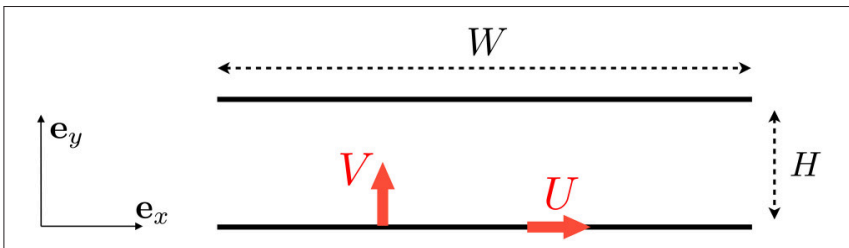

FIGURE 2 | Setup for the flow induced between the tongue and the palate. We denote by $U$ the longitudinal velocity of the tongue (leading a shear flow) and $V$ its vertical upward velocity (leading to a squeeze, pressure-driven flow).

in the transverse (vertical) direct with typical velocity $V$. We use $x$ and $y$ to denote the directions along and perpendicular to the tongue (see Figure 2), with the tongue located at $y=0$ and the palate at $y=H$.

The longitudinal motion leads to a shear flow in the fluid. The solution of Stokes equations in this case is simply $\mathbf{u}=u_{\|} \mathbf{e}_{x}$ with a linear profile [37]

$$
u_{\|}=U \frac{H-y}{H},
$$

where we use the subscript $\|$ to indicate that the flow is driven by motion parallel to the tongue. The typical shear rate acting on the tongue in this case is on the order of $\dot{\gamma}_{\|}=O(U / H)$.

The transverse motion leads to a pressure-driven (squeeze) flow in the thin gap between the tongue and the palate. Assuming unidirectional flow, we have in this case $\mathbf{u}=u_{\perp} \mathbf{e}_{x}$ with $u_{\perp}$ satisfying

$$
\mu \frac{\mathrm{d}^{2} u_{\perp}}{\mathrm{d} y^{2}}=\frac{\mathrm{d} p}{\mathrm{~d} x},
$$

and where we use the subscript $\perp$ to indicate that the flow is driven by motion perpendicular to the tongue. The solution to Equation (2) is the standard parabolic flow

$$
u_{\perp}(y)=\frac{1}{2 \mu} \frac{\mathrm{d} p}{\mathrm{~d} x} y(y-H) .
$$

The pressure gradient is found using conservation of mass. Indeed, the flow rate along the tongue, $Q$, needs to the equal to the flow rate induced by the motion of the tongue, and so $Q=V W$. Integrating Equation (3) across the gap we obtain the scaling

$$
Q \sim-\frac{H^{3}}{\mu} \frac{\mathrm{d} p}{\mathrm{~d} x}
$$

and therefore

$$
\frac{1}{\mu} \frac{\mathrm{d} p}{\mathrm{~d} x} \sim-\frac{V W}{H^{3}},
$$

leading to a flow profile scaling as

$$
u_{\perp}(y) \sim V \frac{W y(H-y)}{H^{3}} .
$$


The typical shear rate on the tongue in this case is therefore given by $\dot{\gamma}_{\perp}=O\left(V W / H^{2}\right)$ and the ratio between the typical longitudinal and transverse shear rates is thus given by

$$
\frac{\dot{\gamma}_{\|}}{\dot{\gamma}_{\perp}} \sim \frac{U / H}{V W / H^{2}} \sim\left(\frac{U}{V}\right)\left(\frac{H}{W}\right) .
$$

Detailed measurements on tongue movement during swallowing shows that $U$ is usually inferior to $V$ and, in the rare cases where it is above, at most a factor of 2 larger [29]. Given that we are in a very high aspect-ratio geometry, $H \ll W$ by about one order of magnitude, and thus we obtain the result that $\dot{\gamma}_{\|} \ll \dot{\gamma}_{\perp}$. The shear flow induced by the tongue in the anterior-posterior direction leads to stresses on the tongue which are smaller in comparison with the stresses induced by the tongue motion in the superior/inferior direction and the resulting squeeze flow. In summary, when a squeeze flow is present, the shear flow is small and can be safely ignored.

\section{DEFORMATION OF SINGLE PAPILLA INTO NEWTONIAN FLOW}

\subsection{Dynamical Regime}

With the Newtonian flow in the tongue characterized in Equations (1) and (6), we now consider its effect on a single papilla. Before computing the shape, and the strain, of a deforming papilla, a number of questions need to be addressed in order to properly characterize their dynamic regime.

First, do papilla deform with unsteady dynamics (transient motion and relaxation) or are the time scales involved sufficiently long that we can model them as deforming in a quasi-steady fashion? This is a question in the realm of fluid-structure interactions of filaments in viscous fluids [38]. With a bending modulus $B$ and a papilla of length $L$ in a viscous fluid of viscosity $\mu$, the typical time scale for the deformation to reach its steadystate shape is given by an elasto-hydrodynamics time scale, $t_{\text {eh }}$, [39]

$$
t_{e h} \sim \frac{\xi L^{4}}{B},
$$

where $\xi$ is the drag coefficient for flow near the papilla, $\xi \approx$ $4 \pi \mu /[\ln (2 L / a)+1 / 2]$. With $\mu=100 \mathrm{mPa} . \mathrm{s}, L \sim 250 \mu \mathrm{m}$, and $B=1.2 \times 10^{-13} \mathrm{~J} . \mathrm{m}$, we obtain a typical time scale of $t_{e h}=$ $0(10 \mathrm{~ms})$. How does this compare with the typical time scale, $T$, for the flow in the mouth? This timescale is given by $H / V$, it is the time scale over which the flow in the mouth is going to change. The vertical velocity is on the order of $V \approx 10 \mathrm{~cm} / \mathrm{s}$ while the thickness is $H \approx 5 \mathrm{~mm}$ and thus $T=H / V \approx 50 \mathrm{~ms}$. Since the high value for $V$ can be considered an upper bound, the time scale we obtained of $50 \mathrm{~ms}$ should be viewed as a lower bound. Given that $T \gtrsim t_{\text {eh }}$ we are thus in a regime where we expect unsteadiness to not play an important role and the deformation will be treated as quasi-static.

The second important question about the dynamics concerns the issue of small vs. large deformations. Do we expect the papillae to deform in the nonlinearly geometric regime where internal tension plays an important role and careful attention needs to be paid to the extensibility of the papilla, or do we remain safely in the linear regime? If we were to remain in the linear regime, the typical deflection of a linear beam would be scaling in the following manner. Let us call $\delta$ the typical magnitude of the papilla tip deflection. For a force of magnitude $q$ per unit length, $\delta$ scales as $\delta \sim q L^{4} / B$ [40]. Here the load is due to the fluid drag and thus $q \sim \xi u$ where we use $u$ to denote the typical velocity of the fluid relative to that of the papilla, leading to $\delta \sim \xi u L^{4} / B=t_{e h} u$.

We need to then consider separately the shear and squeeze flows. In the case of a shear flow, the typical relative velocity around the papilla is $u_{\|} \approx U L / H$ leading to the scaling $\delta / L \sim$ $t_{e h} U / H$. With $t_{e h}=10 \mathrm{~ms}, H=5 \mathrm{~mm}$, and $U \lesssim 10 \mathrm{~cm} / \mathrm{s}$ we obtain $\delta / L=0\left(10^{-1}\right)$ and remain safely in the linear regime. In the case of a squeeze flow we have the scaling near the papilla $u \sim V L W / H^{2}$ and thus we obtain $\delta / L \sim t_{e h} V W / H^{2}$. Here again with $t_{e h}=10 \mathrm{~ms}, H=5 \mathrm{~mm}, V \lesssim 10 \mathrm{~cm} / \mathrm{s}$, and $W=5 \mathrm{~cm}$, we obtain $\delta / L=0(1)$, which is at the limit but is a regime in which the linearized equations of solid mechanics will be at least qualitatively accurate, and approximately quantitatively accurate.

\subsection{Mathematical Model}

In order to describe the deformation of a papilla in a flow, and the elastic strain that it applies to the tongue, we use the formulation for the dynamics of elastic filaments in viscous flows. Let us denote by $\mathbf{r}$ the location of a point along the filament and $s$ the arc length along the filament (from $s=0$ to $s=L$ ). We use $\mathbf{u}$ to denote the external flow field located at position $\mathbf{r}$ and $\tau$ the tension (force per unit length) enforcing inextensibility of the filament. The instantaneous balance of forces and moments on the filament leads then to [38]

$$
\xi\left(\mathbf{r}_{t}-\mathbf{u}\right)=-B \mathbf{r}_{s S S}+\left(\tau \mathbf{r}_{s}\right)_{s} .
$$

In Equation (9) we have used the shorthand notation that $(. . .)_{s}$ means a partial derivative along the $s$ direction and $(. . .)_{t}$ a partial time derivative (so that the first term on the right-hand side of Equation (9) has four derivatives along $s$ ).

To simplify the mathematical approach and the interpretation of the results we proceed to non-dimensionalize the problem. We use the length of the papilla, $L$, as the characteristic length, $t_{e h}$ as the characteristic time (Equation 8), $L / t_{e h}$ as the characteristic velocity and $B / L^{2}$ as characteristic tension. Doing so, Equation (9) becomes

$$
\mathbf{r}_{t}=\mathbf{u}-\mathbf{r}_{s s s s}+\left(\tau \mathbf{r}_{s}\right)_{s}
$$

The equation for the tension $\tau$ is found by enforcing that $\mathbf{r}_{s} \cdot \mathbf{r}_{s}$ remains equal to one for all times, leading to

$$
\tau_{s S}-\tau\left(\mathbf{r}_{s S} \cdot \mathbf{r}_{s S}\right)+4 \mathbf{r}_{s S} \cdot \mathbf{r}_{s S S S}+3 \mathbf{r}_{s S S} \cdot \mathbf{r}_{s S}+\mathbf{r}_{s} \cdot \mathbf{u}_{s}=0 .
$$

In Equation (12) the term including $\mathbf{u}_{s}$ is small and can be neglected since it quantifies gradients of the flow along the length of the papilla. In the linear regime, $\mathbf{r}_{s}=\mathbf{t}=\mathbf{e}_{\mathbf{y}}$ is the tangent to the papilla while $\mathbf{u}_{s}=\partial \mathbf{u} / \partial y$ is the $y$ derivative of the flow, which is nonzero along the $x$ direction only, and therefore $\mathbf{r}_{s} \cdot \mathbf{u}_{s}=0$. We thus obtain the further simplification

$$
\tau_{s s}-\tau\left(\mathbf{r}_{s S} \cdot \mathbf{r}_{s S}\right)+4 \mathbf{r}_{s s} \cdot \mathbf{r}_{s s s}+3 \mathbf{r}_{s S s} \cdot \mathbf{r}_{s s s}=0 .
$$


Furthermore, using our scaling approach from the previous section we can neglect any nonlinear terms in the shape of the papillae. As can be seen in Equation (12), the tension $\tau$ is expected to be quadratic in the filament amplitude and therefore the last term in Equation (10) is cubic, and can be neglected. We thus end up with the linear beam equation

$$
\mathbf{u}-\mathbf{r}_{t}=\mathbf{r}_{s s s},
$$

which, when simplified further due to the fast relaxation of unsteady modes (see Section 4.1), becomes the quasi-steady equation

$$
\mathbf{u}=\mathbf{r}_{s s s s},
$$

Physically, Equation (14) indicates a balance between bending and the drag forces from the fluid.

\subsection{Scaling Laws for Deflections}

Calling $\delta$ the typical deflection of an individual papilla and coming back to dimensional equations, we thus obtain the linearized balance

$$
\xi u \sim B \frac{\mathrm{d}^{4} \delta}{\mathrm{d} y^{4}},
$$

leading to a typical tip deflection on the order of

$$
\delta \sim \frac{\xi L^{4} u}{B}
$$

We can then use the two velocity profiles, Equation (1) and Equation (6), to obtain the typical order of magnitude of the deflections.

\subsubsection{Shear Flow}

For a shear flow we have $u=u_{\|} \sim U L / H$ leding to a typical deflection

$$
\delta_{\|} \sim \frac{\xi U L^{5}}{B H}
$$

The longitudinal strain, $\epsilon$, along the papilla is given by $\epsilon=a \kappa$ where $a$ is the radius of the papilla and $\kappa$ the curvature of the papilla, $\kappa \approx \delta^{\prime \prime}(y) \sim \delta / L^{2}$. The typical longitudinal strain, $\epsilon_{\|}$, scales thus as

$$
\epsilon_{\|} \sim \frac{a \xi U L^{3}}{B H} .
$$

Using the parameters of our model, $\mu=100 \mathrm{mPa} . \mathrm{s}, \xi \approx$ $4 \pi \mu /[\ln (2 L / a)+1 / 2] \approx 0.45$ Pa.s, $L \sim 250 \mu \mathrm{m}, B=1.2 \times$ $10^{-13} \mathrm{~J} . \mathrm{m}, U \approx 10 \mathrm{~cm} / \mathrm{s}, H \approx 5 \mathrm{~mm}$ and $a \approx 50 \mu \mathrm{m}$, we obtain small strains of order $\left|\epsilon_{\|}\right| \approx 6 \%$.

\subsubsection{Squeeze Flow}

In the case of the squeeze flow the velocity scales as

$$
u_{\perp}(y) \sim V \frac{W y(H-y)}{H^{3}} .
$$

Furthermore, since the papillae are much smaller than the mouth, $L \ll H$, the velocity profile close to the tongue is approximately a shear flow and the papilla is not able to feel the curvature of the velocity profile in the center of the mouth. The typical magnitude of the flow near a papilla is therefore given by

$$
u_{\perp}(y) \sim V \frac{W L}{H^{2}}
$$

Using Equation (16), we thus obtain a typical deflection

$$
\delta_{\perp} \sim \frac{\xi V W L^{5}}{B H^{2}}
$$

and therefore a longitudinal strain in the papilla scaling as

$$
\epsilon_{\perp} \sim \frac{a \xi V W L^{3}}{B H^{2}}
$$

Using the same numbers as above with in addition $V \approx 10 \mathrm{~cm} / \mathrm{s}$ and $W=5 \mathrm{~cm}$, leads to a much larger strain than in the longitudinal case, $\left|\epsilon_{\perp}\right| \approx 60 \%$, indicating significant strains. Although a more complete quantitative model would include all nonlinear terms in the beam equation, this results provides a qualitative estimate.

\section{PAPILLAE-INDUCED SUBSTRATE DEFORMATION}

\subsection{Main Question}

We are now in a position to answer the main question of this work: do the papillae, through their deformation and the fact that they are anchored to the tongue, lead to deformation of the tongue which is stronger than if the papillae were not present. In other words, if the mechano-sensors are distributed beneath the surface of the tongue, could papillae amplify deformation of the tongue mediated through fluid-structure interactions? We can quantitatively address this question within our mathematical framework. In addition, since sensory neurons have also been shown to be mechanosensitive themselves (and thus not solely serving conduction of surface mechanosensory cells [41]), it is important to ask whether such neurons could be impacted by papillae strains.

\subsection{Fundamental Solution: Point-Force on an Elastic Substrate}

The first step is to consider the Green's function for the deformation of a semi-infinite elastic substrate due to a point force. With the Green's function known, all other types of deformations can be tackled mathematically.

Consider an elastic medium located in the $x_{3}<0$ semiinfinite plane, as a model for the tongue. The Young's modulus of the tongue is denoted $E$ and its Poisson's ratio $v$. A constant force of magnitude $F_{3}$ is applied at the surface of the tongue, with the surface being parallel to the $\left(x_{1}, x_{2}\right)$ plane. The force is being applied at the origin of the coordinate system. The solution to this Green's function problem is classical [40] and we 
summarize it here. The elastic displacements in the tongue along each direction, $\left\{u_{1}, u_{2}, u_{3}\right\}$ at position $\left\{x_{1}, x_{2}, x_{3}\right\}$ are given by

$$
\begin{aligned}
& u_{1}=\frac{1+\sigma}{2 \pi E}\left[-\frac{x_{1} x_{3}}{r^{3}}-\frac{(1-2 \sigma) x_{1}}{r\left(r-x_{3}\right)}\right] F_{3}, \\
& u_{2}=\frac{1+\sigma}{2 \pi E}\left[-\frac{x_{2} x_{3}}{r^{3}}-\frac{(1-2 \sigma) x_{2}}{r\left(r-x_{3}\right)}\right] F_{3}, \\
& u_{3}=\frac{1+\sigma}{2 \pi E}\left[\frac{x_{3}^{2}}{r^{3}}+\frac{2(1-\sigma)}{r}\right] F_{3},
\end{aligned}
$$

where $r^{2}=\sum_{i} x_{i}^{2}$.

For simplicity we then assume that the tongue is incompressible, meaning its Poisson's ratio is given by $\sigma=1 / 2$. This simplifies the results for the displacements as

$$
\begin{aligned}
& u_{1}=\frac{3}{4 \pi E}\left(-\frac{x_{1} x_{3}}{r^{3}}\right) F_{3}, \\
& u_{2}=\frac{3}{4 \pi E}\left(-\frac{x_{2} x_{3}}{r^{3}}\right) F_{3}, \\
& u_{3}=\frac{3}{4 \pi E}\left(\frac{x_{3}^{2}}{r^{3}}+\frac{1}{r}\right) F_{3} .
\end{aligned}
$$

\subsection{Strain Field}

With the elastic displacements known, we can then compute the strain field, and it is given by the symmetric part of the displacement gradients. As can be seen in Equation (24), the typical displacement inside the tongue scales as $u \sim F / E r$ at a distance $r$ from the point where the force $F$ is being applied. The strains in the tongue, $\varepsilon$, are therefore expected to take the approximate values

$$
\varepsilon \sim \frac{u}{r} \sim \frac{F}{E r^{2}}
$$

\subsection{Point moment on an Elastic Substrate}

The deforming papilla is not applying a force on the tongue but instead it applies a moment at its base. If we denote by $M$ the applied moment then we need to take one additional spatial derivative to obtain the strains in the tongue, leading to

$$
\varepsilon \sim \frac{M}{E r^{3}}
$$

The maximum tongue strain is found near the base of the papilla, $r \sim a$ where $a$ is the radius of the papilla leading to the relevant strain value of

$$
\varepsilon \sim \frac{M}{E a^{3}}
$$

\subsection{Strains in Tongue from Papilla Bending}

The result shown by Equation (27) needs now to be compared with what would happen in the absence of papillae-induced deformation.

\subsubsection{Shear Flow}

In the case of a shear flow, in the absence of papillae the pressure in the Newtonian fluid is constant and equal to its atmospheric value, meaning that the tongue would undergo no forcing normal to its surface. The shear flow would of course lead to shear stresses, but no shear strain in the case of an approximately incompressible tongue. So as far as strain receptors are concerned, they would not measure anything if there were no papillae in this situation.

What is the order of magnitude of the tongue strains, $\varepsilon_{\|}$, we would obtain as induced by the presence of papillae? The moment at the base of an individual papilla scales as $M \sim a F$ with the force $F \sim a^{2} \sigma$ corresponding to typical bending stress $\sigma \sim E_{p} \epsilon$, where $\epsilon$ denotes the longitudinal strain in the papilla and $E_{p}$ the Young's modulus of the papilla. We therefore finally obtain an applied moment $M \sim a^{3} E_{p} \epsilon$.

We saw in Equation (18) that in the case of a shear flow we have a longitudinal strain inside the papilla

$$
\epsilon_{\|} \sim \frac{a \xi U L^{3}}{B H},
$$

and thus the moment applied at the base of the papilla on the tongue due to shear-induced bending is given by

$$
M \sim \frac{\xi a^{4} E_{p} U L^{3}}{B H} .
$$

Using the scaling relationship between the Young's modulus of the papilla and the bending rigidity $B \sim a^{4} E_{p}$, we obtain a simpler formula for the torque magnitude as

$$
M \sim \frac{\xi U L^{3}}{H} .
$$

With this value for the moment, we can then use Equation (27) to find the maximum elastic strains in the tongue

$$
\varepsilon_{\|} \sim \frac{\xi U L^{3}}{a^{3} E H}
$$

Provided that the Young's modulus of the tongue is similar to that of individual papillae, which as a first approximation it should, then writing Equation (31), we recognize the elastohydrodynamics time scale, $t_{e h}$, from Equation (8), as a function of

$$
\varepsilon_{\|} \sim t_{e h} \frac{a U}{L H}
$$

Putting in numbers, we recall that $t_{e h}=10 \mathrm{~ms}, U=10 \mathrm{~cm} / \mathrm{s}$, $L=250 \mu \mathrm{m} a=50 \mu \mathrm{m}, E=25 \mathrm{kPa}$, and $H=5 \mathrm{~mm}$. This leads to $t_{\text {eh }} \approx 3 \mathrm{~ms}$ and gives a maximum tongue strain of

$$
\varepsilon_{\|} \sim 4 \% .
$$

Thus in shear flow we propose the advantage of having papillae distributed on the tongue: they create a normal mechanical deformation of the tongue which would not take place in their absence. 


\subsubsection{Squeeze Flow}

In the case of a shear flow, the scalings are different. As in previous cases discussed, we expect to get an even stronger effect than in the longitudinal situation.

From Equation (22), the magnitude of the elongational strain in the papilla due to its bending is given by

$$
\epsilon_{\perp} \sim \frac{a \xi V W L^{3}}{B H^{2}}
$$

and therefore the torque applied at the base of the papilla on the tongue has a typical magnitude

$$
M \sim \frac{\xi a^{4} E_{p} V W L^{3}}{B H^{2}} \sim \frac{\xi V W L^{3}}{H^{2}} .
$$

With this, the typical strains in the tongue in the case of a squeeze flow, $\varepsilon_{\perp}$, are

$$
\varepsilon_{\perp} \sim \frac{\xi V W L^{3}}{a^{3} E H^{2}} .
$$

Comparing Equations (31) and (36) we find the ratio

$$
\frac{\varepsilon_{\perp}}{\varepsilon_{\|}} \sim \frac{V W}{U H} .
$$

Using the same numbers as above except $W=5 \mathrm{~cm}, V=$ $10 \mathrm{~cm} / \mathrm{s}$, this leads to a significantly larger

$$
\varepsilon_{\perp} \sim 40 \%
$$

This result needs to be compared with what would happen in the absence of papillae. In that case the elastic stress in the tongue would be uniform everywhere and equal to the fluid pressure. Approximately, this hydrodynamic pressure is given by

$$
p \sim \frac{\mu V W^{2}}{H^{3}}
$$

and thus the strains in the tongue in the absence of papillae, $\varepsilon_{p}$, would be

$$
\varepsilon_{p} \sim \frac{p}{E} \sim \frac{\mu V W^{2}}{E H^{3}}
$$

leading to

$$
\frac{\varepsilon_{p}}{\varepsilon_{\perp}} \sim \frac{\mu}{\xi} \frac{W}{H}\left(\frac{a}{L}\right)^{3}
$$

\section{REFERENCES}

1. Burbidge A, Le Révérend B. Biophysics of food perception. J Phys D Appl Phys. (2016) 49:114001. doi: 10.1088/0022-3727/49/11/114001

2. Chandrashekar J, Hoon MA, Ryba NJP, Zuker CS. The receptors and cells for mammalian taste. Nature (2006) 444:288-94. doi: 10.1038/nature 05401
With $W=5 \mathrm{~cm}, L=250 \mu \mathrm{m}, a=50 \mu \mathrm{m}, H=5 \mathrm{~mm}$ this leads to

$$
\frac{\varepsilon_{p}}{\varepsilon_{\perp}} \sim 0.02
$$

so the presence of papillae leads to an increase of two orders of magnitude of the strains in the tongue.

\section{CONCLUSION}

The analytical and numerical solutions derived in this article confirm that the topology of the tongue can offer mechanical advantage for texture perception. This effect is dependent on the presence and location of mechanosensitive cells and neurons in relation to such topological structures. This, to the best of our knowledge, remains to be studied, however recent work reporting corpuscular endings in mice filiform papillae indicates locations consistent with the mechanism described here [9].

Most interestingly, we prove that depending on the putative location of those mechanosensitive cells, filiform papillae may either serve sensing purposes in a direct fashion (papillae strains), similarly to superficial neuromasts or in an indirect fashion (tongue strains). In the latter, instead of sensing strains themselves, papillae might encode stresses by inducing strains in the tongue which then lead to bulk sensing. The underlying neurophysiology for those two cases would be dramatically different in terms of strain orientation and magnitude. The application from the developed model could then be used to define the levels of stress and strain that are experienced by different cells depending on the physiological location.

One can also speculate that similarly to the skin, the tongue is populated by various types of cellular mechanosensors, encoding information from different locations and responding to different levels of stresses and frequencies. In this case both direct and indirect sensing may occur from the filiform papillae bending.

As a final perspective, since we have argued that the aspect ratio of filiform papillae is similar to that of other biological structures known to support external stress field encoding, we propose that models similar to ours will also be applicable to such structures. We hope that biologists and biophysicists will be able to apply these ideas to other biological systems.

\section{AUTHOR CONTRIBUTIONS}

$\mathrm{BL}$ and $\mathrm{CP}$ designed the project; EL derived the analytical model; $\mathrm{EL}, \mathrm{CP}$, and $\mathrm{BL}$ wrote the article.
3. Ishida Y, Ugawa S, Ueda T, Murakami S, Shimada S. Vanilloid receptor subtype-1 (VR1) is specifically localized to taste papillae. Mol Brain Res. (2002) 107:17-22. doi: 10.1016/S0169-328X(02)00441-2

4. Dhaka A, Earley TJ, Watson J, Patapoutian A. Visualizing cold spots: TRPM8expressing sensory neurons and their projections. J Neurosci. (2008) 28:56675. doi: 10.1523/JNEUROSCI.3976-07.2008

5. Scott Blair GW. Measurements of Mind and Matter (1950). 
6. de Wijk RA, Prinz JF, Engelen L, Weenen H. The role of $\alpha$-amylase in the perception of oral texture and flavour in custards. Phys Behav. (2004) 83:81-91. doi: 10.1016/j.physbeh.2004.07.014

7. Peyron MA, Mishellany A, Woda A. Particle size distribution of food boluses after mastication of six natural foods. J Dental Res. (2004) 83:578-82. doi: $10.1177 / 154405910408300713$

8. Engelen L, van der Bilt A, Schipper M, Bosman F. Oral size perception of particles: effect of size, type, viscosity and method. J Text Stud. (2005) 36:373-86. doi: 10.1111/j.1745-4603.2005.00022.x

9. Moayedi Y, Duenas-Bianchi L, Michlig S, le Coutre J, Le Révérend B, Lumpkin E. Somatosensory contribution to food oral processing. In: Proceedings of the 4th Food Oral Processing Conference. Lausanne (2016).

10. Sato O, Maeda T, Kobayashi S, Iwanaga T, Fujita T. Filiform papillae as a sensory apparatus in the tongue: an immunohistochemical study of nervous elements by use of neurofilament protein (NFP) and S-100 protein antibodies. Cell Tissue Res. (1988) 252:231-8. doi: 10.1007/BF00 214365

11. Malone AMD, Anderson CT, Tummala P, Kwon RY, Johnston TR, Stearns T, et al. Primary cilia mediate mechanosensing in bone cells by a calciumindependent mechanism. Proc Natl Acad Sci USA. (2007) 104:13325-30. doi: 10.1073/pnas.0700636104

12. McHenry MJ, van Netten SM. The flexural stiffness of superficial neuromasts in the zebrafish (Danio rerio) lateral line. J Exp Biol. (2007) 210:4244-53. doi: 10.1242/jeb.009290

13. Triantafyllou MS, Weymouth GD, Miao J. Biomimetic survival hydrodynamics and flow sensing. Annu Rev Fluid Mech. (2016) 48:1-24. doi: 10.1146/annurev-fluid-122414-034329

14. Baratz RS, Farbman AI. Morphogenesis of rat lingual filiform papillae. Am J Anat. (1975) 143:283-301. doi: 10.1002/aja.1001430303

15. Nagato T, Nagaki M, Murakami M, Tanioka H. Morphological studies of rat lingual filiform papillae. Okajimas Folia Anat Jpn. (1989) 66:195-209. doi: 10.2535/ofaj1936.66.4_195

16. Toprak B. Light and scanning microscopic structure of filiform papillae in mice. Veterinarski Arhiv. (2007) 76:555. Available online at: http://hrcak.srce. $\mathrm{hr} / 22261$

17. Karan M, Yilmaz S, Aydin A. Morphology of the filiform lingual papillae in porcupine (Hystrix cristata). Anat Histol Embryol. (2011) 40:100-3. doi: 10.1111/j.1439-0264.2010.01045.x

18. Chamorro CA, Fernandez JG, Paz P, Pelaez B, Anel L. Scanning electron microscopy of the wild boar and pig lingual papillae. Histol Histopathol. (1994) 9:657-67.

19. Toyoda M, Sakita S, Kagoura M, Morohashi M. Electron microscopic characterization of filiform papillae in the normal human tongue. Arch Hist Cytol. (1998) 61:253-68. doi: 10.1679/aohc.61.253

20. Manabe M, Lim HW, Winzer M, Loomis CA. Architectural organization of filiform papillae in normal and black hairy tongue epithelium: dissection of differentiation pathways in a complex human epithelium according to their patterns of keratin expression. Arch Dermat. (1999) 135:177-81. doi: 10.1001/archderm.135.2.177

21. Iwasaki S. Evolution of the structure and function of the vertebrate tongue. $J$ Anat. (2002) 201:1-13. doi: 10.1046/j.1469-7580.2002.00073.x

22. Yamashita K, OdDalkhsuren S. The papillae of the human tongue. In: Kat H, Shimizu T, editors. Tongue: Anatomy, Kinematics and Diseases. Hauppauge, NY: Nova Science Publishers, Inc. (2012). pp. 143-54.

23. Ranc H, Servais C, Chauvy PF, Debaud S, Mischler S. Effect of surface structure on frictional behaviour of a tongue/palate tribological system. Trib Int. (2006) 39:1518-26. doi: 10.1016/j.triboint.2006.01.017

24. Dresselhuis DM, De Hoog EHA, Cohen Stuart MA, Van Aken GA. Application of oral tissue in tribological measurements in an emulsion perception context. Food Hydrocoll. (2008) 22:323-35. doi: 10.1016/j.foodhyd.2006.12.008

25. Kamm R, Lammerding J, Mofrad M. Cellular nanomechanics. In: Bhushan B, editor. Springer Handbook of Nanotechnology. New York, NY: Springer (2010). pp. 1171-200.

26. Berdyyeva TK, Woodworth CD, Sokolov I. Human epithelial cells increase their rigidity with ageing in vitro: direct measurements. Phys Med Biol. (2005) 50:81. doi: 10.1088/0031-9155/50/1/007

27. Spassova I. Ultrastructure of the simple encapsulated nerve endings (simple end-bulbs of Krause) in the tongue of the cat. J Anat. (1974) 118(Pt 1):1-9.

28. Lumpkin EA, Caterina MJ. Mechanisms of sensory transduction in the skin. Nature (2007) 445:858-65. doi: 10.1038/nature 05662

29. Shawker TH, Sonies B, Stone M, Baum BJ. Real-time ultrasound visualization of tongue movement during swallowing. J Clin Ultrasound (1983) 11:485-90. doi: 10.1002/jcu.1870110906

30. Kristensen D, Jensen PY, Madsen F, Birdi KS. Rheology and surface tension of selected processed dairy fluids: influence of temperature. J Dairy Sci. (1997) 80:2282-90. doi: 10.3168/jds.S0022-0302(97)76177-0

31. Leighton A, Williams OE. The effect of temperature on the basic viscosity of ice-cream mixes. J Phys Chem. (1927) 31:1663-8. doi: 10.1021/j150281a004

32. Adapa S, Dingeldein H, Schmidt KA, Herald TJ. Rheological properties of ice cream mixes and frozen ice creams containing fat and fat replacers. J Dairy Sci. (2000) 83:2224-9. doi: 10.3168/jds.S0022-0302(00)75106-X

33. Muse MR, Hartel RW. Ice cream structural elements that affect melting rate and hardness. J Dairy Sci. (2004) 87:1-10. doi: 10.3168/jds.S00220302(04)73135-5

34. Nicosia MA, Robbins J. The fluid mechanics of bolus ejection from the oral cavity. J Biomech. (2001) 34:1537-44. doi: 10.1016/S0021-9290(01)00147-6

35. Kim S, Karilla JS. Microhydrodynamics: Principles and Selected Applications. Boston, MA: Butterworth-Heinemann (1991).

36. Happel J, Brenner H. Low Reynolds Number Hydrodynamics. Englewood Cliffs, NJ: Prentice Hall (1965).

37. Batchelor GK. An Introduction to Fluid Dynamics. Cambridge, UK: Cambridge University Press (1967).

38. Powers TR. Dynamics of filaments and membranes in a viscous fluid. Rev Mod Phys. (2010) 82:1607-31. doi: 10.1103/RevModPhys.82.1607

39. Wiggins $\mathrm{CH}$, Goldstein RE. Flexive and propulsive dynamics of elastica at low Reynolds number. Phys Rev Lett. (1998) 80:3879-82. doi: 10.1103/PhysRevLett.80.3879

40. Landau LD, Lifshitz EM. Theory of Elasticity. 3rd Edn. Oxford: Pergamon Press (1986).

41. Woo SH, Ranade S, Weyer AD, Dubin AE, Baba Y, Qiu Z, et al. Piezo2 is required for Merkel-cell mechanotransduction. Nature (2014) 509:622-6. doi: 10.1038 /nature 13251

Conflict of Interest Statement: The authors declare that the research was conducted in the absence of any commercial or financial relationships that could be construed as a potential conflict of interest.

BL and CP are employees of the Nestlé Research Center. EL was consulting with Nestlé Research Center during the study.

Copyright (c) 2016 Lauga, Pipe and Le Révérend. This is an open-access article distributed under the terms of the Creative Commons Attribution License (CC BY). The use, distribution or reproduction in other forums is permitted, provided the original author(s) or licensor are credited and that the original publication in this journal is cited, in accordance with accepted academic practice. No use, distribution or reproduction is permitted which does not comply with these terms. 